\title{
'Candidatus Paenicardinium endonii', an endosymbiont of the plant-parasitic nematode Heterodera glycines (Nemata: Tylenchida), affiliated to the phylum Bacteroidetes
}

\author{
Gregory R. Noel ${ }^{1}$ and Ndeme Atibalentja ${ }^{2}$ \\ US Department of Agriculture, Agricultural Research Service ${ }^{1}$ and Department of Crop \\ Sciences, Turner Hall, University of Illinois², Urbana, IL 61801, USA
}

Correspondence

Gregory R. Noel

g-noel1@uiuc.edu

\begin{abstract}
Bacteria-like endosymbionts of females of the plant-parasitic nematodes Globodera rostochiensis and Heterodera goettingiana and juveniles of Heterodera glycines were first observed during transmission electron microscopy (TEM) studies conducted in the 1970s. These organisms were characterized as being rod-shaped, ranging in size from 0.3 to $0.5 \mu \mathrm{m}$ in diameter and 1.8 to $3 \mu \mathrm{m}$ in length and containing structures labelled as striated inclusion bodies or tubular structures.

A population of $\mathrm{H}$. glycines was obtained from the soybean field where infected nematodes were first discovered in order to conduct TEM studies of females and males and to determine the phylogenetic position of the $H$. glycines endosymbiont among bacteria by studying the 16S rRNA and gyrB gene sequences. The bacterium was observed in the pseudocoelom and intestine of juveniles, females and males, in hypodermal chords of juveniles and males, in ovary walls and in oocytes and spermatozoa. The bacterium was polymorphic, measuring $0.4-0.8 \times 2.5-4.5 \mu \mathrm{m}$, and many specimens contained an array of microfilament-like structures similar to those observed in 'Candidatus Cardinium hertigii', the endosymbiont of Encarsia spp. wasps. Phylogenetic analysis of the $16 \mathrm{~S}$ rRNA and gyrB genes of the $H$. glycines-infecting bacterium revealed $93 \%$ and $81 \%$ sequence identity, respectively, to the homologous genes in 'Candidatus $\mathrm{C}$. hertigii'. Thus, the name 'Candidatus Paenicardinium endonii' is proposed for the bacterial endosymbiont of the plant-parasitic nematode $H$. glycines.
\end{abstract}

\section{INTRODUCTION}

The occurrence of bacterial endosymbionts in invertebrates is well documented and, among the endosymbionts of nematodes, the genus Wolbachia is perhaps the most studied (Taylor, 2003). Wolbachia species are associated with the animal-parasitic nematodes Brugia malayi and Wucheria bancrofti (both Spiruida: Filariidae), which cause lymphatic filariasis and are vectored by mosquitoes, and Onchocerca volvulus (Spiruida: Onchocercidae), which causes onchocerciasis or river blindness and is vectored by black flies (Taylor, 2003; Saint André et al., 2002). Wolbachia species are believed to be the primary cause of acute inflammatory filarial disease and the inflammatory response of the cornea.

Abbreviation: MLS, microfilament-like structure.

The GenBank/EMBL/DDBJ accession numbers for the 16S rRNA gene and gyrB sequences of 'Candidatus Paenicardinium endonii' are DO314214 and DO314215.

Maximum-likelihood trees based on 16S rRNA gene and gyrB sequences showing the position of 'Candidatus Paenicardinium endonii' within the Bacteroidetes are available as supplementary material in IJSEM Online.
Members of the nematode genera Steinernema and Heterorhabditis have a mutualistic association with endosymbiotic bacteria of the genus Xenorhabdus in which the nematode host vectors the bacterium into the haemocoel of the insect host, where the bacterium is deposited in the haemolymph, multiplies and kills the insect by inducing septicaemia (Kaya, 1993). Another group of nematode endosymbionts includes three candidate verrucomicrobial species of 'Candidatus Xiphinematobacter' associated with three species of plant-parasitic nematodes of the genus Xiphinema (Dorylaimida: Longidoridae) (Vandekerckhove et al., 2000). These nematodes reproduce by thelytokous parthenogenesis and the 'Candidatus Xiphinematobacter' organisms are inherited maternally. Another group of bacterial endosymbionts, which have characteristic microfilament-like structures (MLSs) similar to those of 'Candidatus Cardinium hertigii', infect the cyst-forming, plant-parasitic nematodes Globodera rostochiensis (potato cyst nematode), Heterodera glycines (soybean cyst nematode) and Heterodera goettingiana (pea cyst nematode) (all Tylenchida: Heteroderidae). These endosymbionts were first observed in females and males of two populations of G. rostochiensis 
and one population of $H$. goettingiana during transmission electron microscopy (TEM) studies (Shepherd et al., 1973) and subsequently in H. glycines juveniles (Endo, 1979). The endosymbionts have not been encountered in other populations of these species or in other genera of nematodes. Unlike their allies that infect arthropods (Zchori-Fein et al., 2004), the cyst-nematode-infecting endosymbionts are not known to affect host preference or reproduction or to cause other pathological manifestations (Shepherd et al., 1973).

The objectives of our study were twofold. The first was to confirm observations of infection of second-stage juveniles (J2) of $\mathrm{H}$. glycines and to identify sites of infection of the endosymbiont in females and males. The second objective was to characterize the endosymbiont and to determine its phylogenetic position within the bacterial domain by using $16 \mathrm{~S}$ rRNA and $g y r B$ gene sequences.

\section{METHODS}

Culture of $\boldsymbol{H}$. glycines. Soil infested with $H$. glycines was obtained from the Ames Plantation near Grand Junction, TN, USA. This population was obtained from the same field as the population studied by Endo (1979). Cysts (dead bodies of females containing eggs) were extracted from soil and added to pots containing steamsterilized sand in which soybean seeds were planted. The cultures have been maintained in a greenhouse for many years by repeating the extraction and replanting process as needed.

Electron microscopy. Male nematodes were obtained from infected soybean roots placed in a mist chamber. Vigorous individuals were selected with the aid of a dissecting microscope and placed in distilled water in a BPI dish. The water was drawn off with a micropipette and replaced with cold $2 \%$ glutaraldehyde in $0.08 \mathrm{M}$ sodium phosphate buffer, $\mathrm{pH} 7 \cdot 2$. Nematodes were cut in half, fixed for 1 week and rinsed with buffer. Heads and tails were placed in separate vials and post-fixed overnight in $1 \%$ osmium tetroxide. Sections were selected individually, placed in triangular slits of excised agar approximately the size of the tip of a beam capsule and then covered with warm agar. Agar blocks containing nematode pieces were excised, dehydrated in an ethanol series $(10,25,50,75$, 95 and $100 \%$ ) and placed in a $50 \%$ solution of ethanol and n-butyl glycidyl ether (BGE) for $1 \mathrm{~h}$. The $50 \%$ solution was replaced with $100 \%$ BGE and held overnight under vacuum. The sections were infiltrated under vacuum for several hours with 50, 75 and $100 \%$ Quetol 654 (Electron Microscopy Sciences). Specimens were placed in fresh $100 \%$ Quetol 654 and infiltrated overnight under vacuum and then embedded in Quetol 654 for $72 \mathrm{~h}$ at $70^{\circ} \mathrm{C}$. Females and $\mathrm{J} 2$ were prepared as for males except that nematodes were not cut. Ultrathin sections $(90-100 \mathrm{~nm})$ were cut with an ultramicrotome (Reichert) and mounted on uncoated grids. Sections were stained with aqueous saturated uranyl acetate for $40 \mathrm{~min}$ and with Sato's triple lead stain (Hanaichi et al., 1986) for $1 \mathrm{~min}$. Sections were observed and photographed with a Hitachi H-600 transmission electron microscope.

PCR amplification, cloning and sequencing of the 16S rRNA and $\boldsymbol{g y r} \boldsymbol{B}$ genes. J2 presumably infected with the bacterial endosymbiont were hatched from eggs and rinsed by repeated centrifugation in sterile water. Nematodes were pipetted onto a column of sterile, moist silica and allowed to migrate through the 5-cm-long column into a reservoir of sterile water. Nematodes were concentrated by centrifugation under sterile conditions and used for extraction of DNA as described by Atibalentja et al. (2004). Briefly, several thousand nematodes in $200 \mu \mathrm{l}$ sterile water were added to an equal volume of acid-washed $0 \cdot 150-0 \cdot 212 \mathrm{~mm}$ diameter glass beads (Sigma-Aldrich) in a screw-capped vial and subjected to vibration in a Mini-BeadBeater (BiosPec Products) at 5000 r.p.m. for $2 \mathrm{~min}$. The nematode plus silica slurry was centrifuged at $16000 \mathrm{~g}$ for $5 \mathrm{~min}$ and the supernatant was transferred into a $1.6 \mathrm{ml}$ microfuge tube and stored at $-20^{\circ} \mathrm{C}$. Combinations of universal primers $27 \mathrm{f} / 1492 \mathrm{r}$ (Atibalentja et al., 2004; Lane, 1991) and UP-1/UP-2r (Yamamoto \& Harayama, 1995) were used for PCR amplification of the 16S rRNA and $\operatorname{gyr} B$ genes, respectively, following procedures described previously (Atibalentja et al., 2004), except that the annealing temperature for the $\operatorname{gyrB}$ gene was set at $56^{\circ} \mathrm{C}$. Cloning and sequencing of PCR products were also conducted as reported previously (Atibalentja et al., 2004). Both DNA strands were sequenced from each of three and four clones, respectively, for the 16S rRNA and gyrB genes, and the resulting DNA fragments were assembled with the computer program SEQUENCHER (Gene Codes).

Phylogenetic analysis. The consensus DNA sequences for the $16 \mathrm{~S}$ rRNA and $\operatorname{gyr} B$ genes were subjected to sequence-similarity searches, using the BLAST algorithm (Altschul et al., 1997). The ORF finder utility of GenBank also was used to identify the putative open reading frame of the $g y r B$ gene, and the deduced amino acid sequence was also submitted to BLAST. Homologous sequences from a selection of bacteria suggested by the BLAST results were retrieved from public databases and aligned with the corresponding sequence from the endosymbiont of $H$. glycines, using CLUSTAL X (Thompson et al., 1997). The alignments were edited manually to remove terminal and internal gap-rich regions. Phylogenetic analyses were conducted with PAUP ${ }^{*} 4.0$ b10 (Swofford, 2003) under the maximum-parsimony (MP), maximum-likelihood (ML) and distance optimality criteria, following the procedures described by Zchori-Fein et al. (2004) unless indicated otherwise. Specifically, the HKY85 distance and the weighted least-squares objective function were used to construct the neighbour-joining $(\mathrm{NJ})$ tree, which was used to estimate the parameters for ML reconstruction under a general time reversible (GTR) model of nucleotide substitution, with rate heterogeneity among sites $(\Gamma)$ and a given proportion of invariable sites (I). The robustness of the inferred clades was assessed through bootstrap analysis, which, for MP and NJ trees, involved 10000 replicates (of 100 random-addition sequence replicates each for MP). For ML trees, bootstrap analysis involved 1000 replicates of 10 random-addition sequence replicates each for the 16S RNA gene and 100 replicates of one random-addition sequence replicate each for the gyrB gene.

We also sought to identify signature sequences for the 16S rRNA gene of the $H$. glycines endosymbiont, using a slight modification of the method described by Zchori-Fein et al. (2004). Briefly, candidate oligonucleotides suggested by MacClade (Maddison \& Maddison, 2001) were submitted to probe match in the Ribosomal Database Project (RDP II Release 9, http://rdp.cme.msu.edu/html) (Cole et al., 2003) and to BLAST search for short, nearly exact matches in GenBank only after they were selected by PRIMROSE (Ashelford et al., 2002) as oligonucleotide probes that exclusively targeted the 16S rRNA gene sequence of the $H$. glycines endosymbiont.

\section{RESULTS AND DISCUSSION}

\section{Morphology}

The endosymbiont was observed in the pseudocoelom of $\mathrm{J} 2$, females and males. Additionally, bacteria were observed in oocytes, ovaries, spermatozoa and the intestine of both genders (Fig. 1). For the most part, our observations confirmed those of Shepherd et al. (1973), who reported 

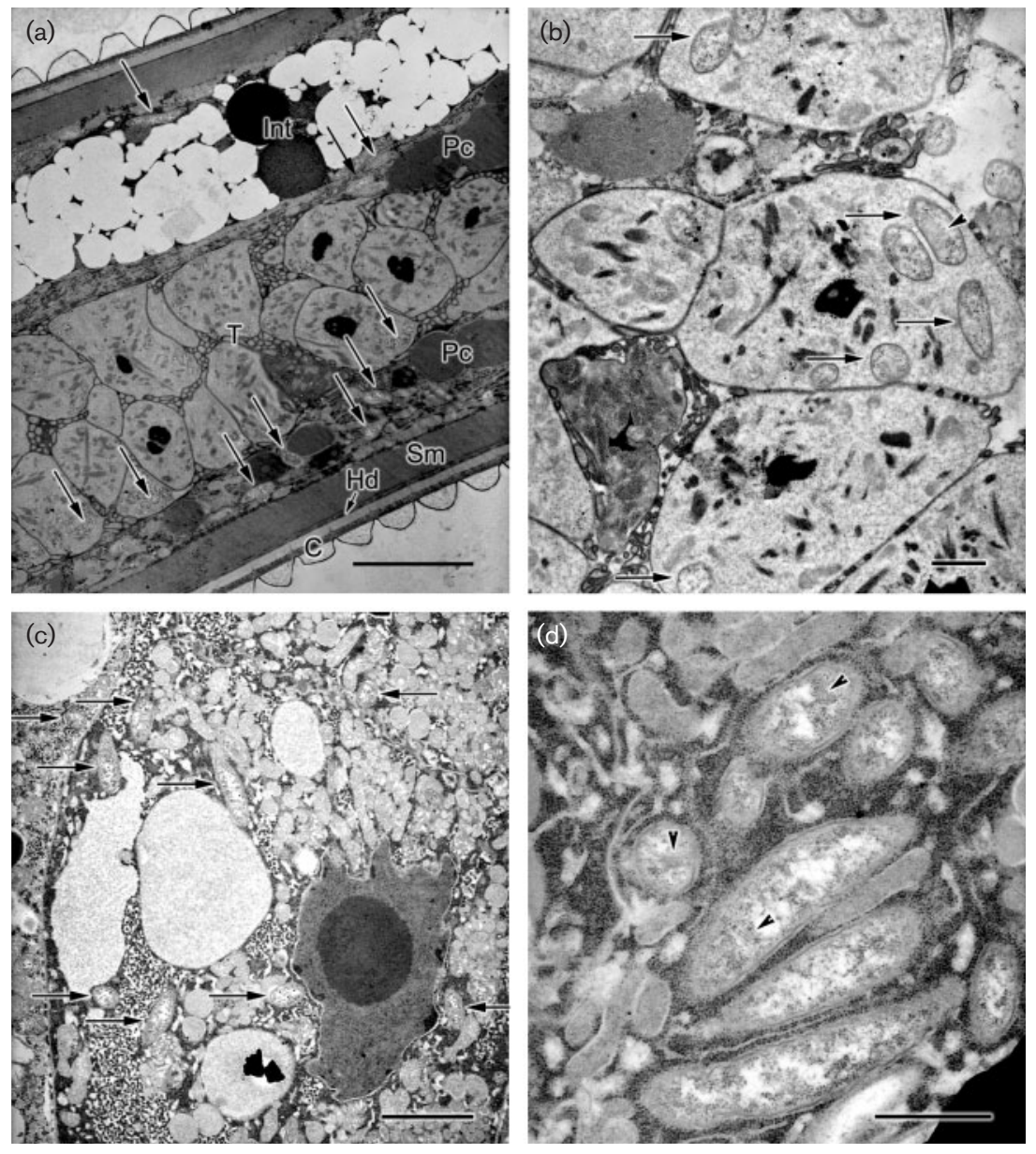

Fig. 1. Electron micrographs of 'Candidatus Paenicardinium endonii' in $\mathrm{H}$. glycines males and females. (a) Longitudinal section of a male in which the presence of bacteria in the intestine (Int), pseudocoelom $\left(\mathrm{Pc}_{\mathrm{C}}\right)$ and spermatozoa within a testis $(T)$ is indicated by arrows. The bacterium was not observed in somatic muscles $(\mathrm{Sm})$, the hypodermis $(\mathrm{Hd})$ or the cuticle (C). (b) Higher magnification of bacteria (arrows) in spermatozoa. MLSs are indicated by arrowheads. (c) Presence of bacteria in ovary and oocytes is indicated by arrows. (d) Bacteria in pseudocoelom of female. MLSs are indicated by arrowheads. Bars, $5 \mu \mathrm{m}$ (a), $1 \mu \mathrm{m}$ (b, d) and $3 \mu \mathrm{m}$ (c). that the bacterium was most common in reproductive tissues of females including the ovary, oviduct wall and oocytes. We did not observe bacteria in the hypodermis of females, but found them in the hypodermal chords of males. Our study of $\mathrm{J} 2$ was done to confirm the presence of the bacterium in the second-stage infective juveniles. Bacteria were observed in the hypodermal chords, intestinal tissue and pseudocoelom. The research of Endo (1979) was an exhaustive study of the oesophageal region of infective juveniles. Bacteria observed in that study were most abundant in the sarcoplasm of stylet protractor muscles and muscles of the median bulb and the oesophageal glands. In our study, the bacterium was polymorphic in appearance due to the random orientation of individuals within nematode tissues and cells and sectioning of bacteria that often was tangential, especially along the length of the organisms. We found that the diameter of the bacteria ranged from $0 \cdot 4$ to $0 \cdot 8 \mu \mathrm{m}$ and the length ranged from $2 \cdot 5$ to $4 \cdot 5 \mu \mathrm{m}$. These values are similar to those reported by Endo (1979), but greater than the values reported by Shepherd et al. (1973) for the endosymbionts in the nematodes $G$. rostochiensis and H. goettingiana and for 'Candidatus C. hertigii' (Zchori-Fein et al., 2004). MLSs were not observed in all bacteria (Fig. 2). It is unclear whether they were absent or in a plane or section that was not studied (Zchori-Fein et al., 2004). In our study and previous studies, some MLSs extended across the width of the cell, whereas some extended part way. The width of MLSs that we determined $(17 \mathrm{~nm})$ was similar to that of Endo (1979), but wider than the 14-15 nm reported by Shepherd et al. (1973). These differences might be due to artefacts of fixation and embedding or calibration of instruments. We observed what appeared to be bundles of MLSs in cross section (Fig. 2). However, cell walls were not apparent with these structures. Endo (1979) observed a cross section of MLSs inside a cell with a clearly defined cell wall.

\section{Sequence and phylogenetic analysis}

16S rRNA gene. PCR amplification, using the universal primers $27 \mathrm{f}$ and $1492 \mathrm{r}$, of crude DNA extracts prepared from $H$. glycines $\mathrm{J} 2$ harbouring the unidentified bacterial endosymbiont resulted in a single band of the expected size $(1.5 \mathrm{~kb})$. Cloning and sequencing of the gel-eluted DNA fragment yielded a 1490 bp DNA sequence $(G+C$ content $50 \cdot 1 \mathrm{~mol} \%$ ) that shared $94 \%$ nucleotide identity with the 16S rRNA gene of the endosymbiont of Metaseiulus occidentalis (GenBank accession no. AY635291), the best fit returned by BLAST searches, and $93 \%$ identity with 'Candidatus C. hertigii' (accession no. AY331187). The alignment of $16 \mathrm{~S}$ rRNA gene sequences used to infer 


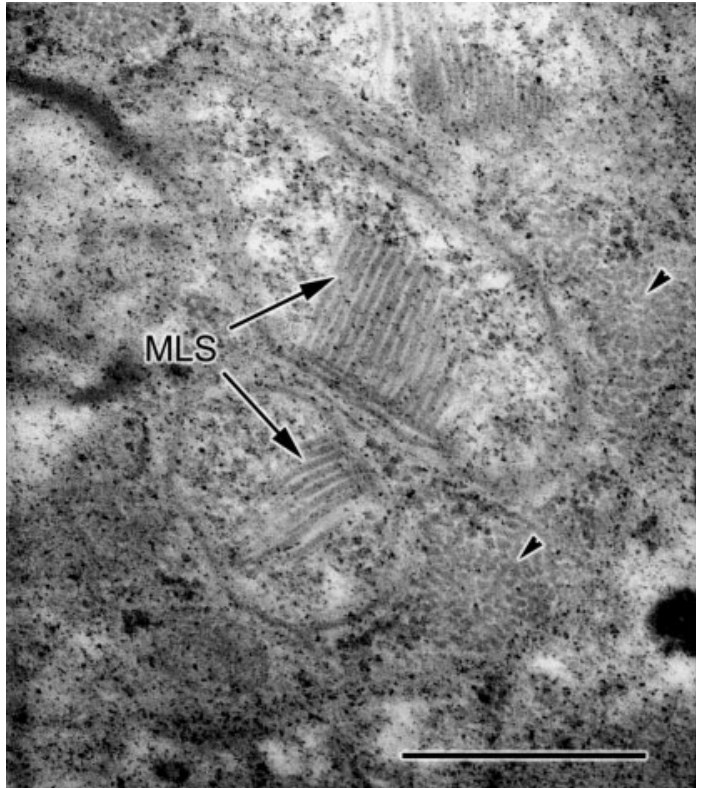

Fig. 2. Electron micrograph of 'Candidatus Paenicardinium endonii' in an $H$. glycines male. MLSs are indicated by arrows. Two possible cross sections of MLSs appear as bundles of microfilaments (arrowheads). Bar, $0.5 \mu \mathrm{m}$.

the phylogeny of the $H$. glycines endosymbiont included 12 taxa and 1467 characters, 1208 of which were constant and 173 of which were parsimony-informative. MP analysis produced two most-parsimonious trees, each 369 steps in length (consistency index 0.79). The two MP trees placed the $H$. glycines endosymbiont in a lineage that had diverged much earlier from the common ancestor of acari and insect endosymbionts, a finding that was also supported by the NJ and ML trees (Fig. 3 and Supplementary
Fig. S1 available in IJSEM Online). Using the NJ tree under the ML criterion, the nucleotide substitution parameters were estimated as follows: $A-C, 1 \cdot 96 ; A-G, 7 \cdot 26$; $\mathrm{A}-\mathrm{T}, 1 \cdot 33 ; \mathrm{C}-\mathrm{G}, 0 \cdot 08 ; \mathrm{C}-\mathrm{T}, 10 \cdot 25$; and $\mathrm{G}-\mathrm{T}, 1 \cdot 00$. The proportion of invariant sites (I) was estimated at 0.56 and the shape parameter $(\alpha)$ of the gamma distribution was equal to $0 \cdot 71$.

Of the two oligonucleotide sequences previously reported as unique to 'Candidatus Cardinium' (Zchori-Fein et al., 2004), only one, $5^{\prime}$-GGTCTTTAACTGACGCT-3', is present in the $16 \mathrm{~S}$ rRNA gene sequence of the $H$. glycines endosymbiont (position 728). This oligonucleotide did not meet the PRIMROSE selection criteria for a probe (Ashelford et al., 2002). In contrast, we have identified two oligonucleotide sequences, 5' -CGCGATACAATCGTGTGTGTCC-3' (position 822) and $5^{\prime}$-CATCCCTAGAAATAGGGAGTTCCGAAA-3' (position 991), that are unique to the H. glycines endosymbiont.

gyrB gene. A single band of the expected size also was obtained when DNA extracts from $H$. glycines J2 carrying the unidentified bacterium were amplified by PCR using the primers UP-1 and UP-2r. Cloning and sequencing of the resulting DNA fragment produced a $1237 \mathrm{bp}$ sequence $(\mathrm{G}+\mathrm{C}$ content $41.7 \mathrm{~mol} \%)$ that contained a 397 aa partial ORF. BLAST searches using the DNA sequence or the deduced amino acid sequence of the partial ORF retrieved the gyrB gene of the 'Candidatus Cardinium' endosymbiont of Encarsia pergandiella (GenBank accession nos. AY332002 and AAQ98790) as the best match with the query sequence, sharing 83 and $81 \%$ nucleotide and amino acid identity, respectively. Phylogenetic analyses using either the nucleotide or the amino acid sequence of the $\operatorname{gyrB}$ gene produced similar results for the position of the $H$. glycines endosymbiont. Therefore, the following

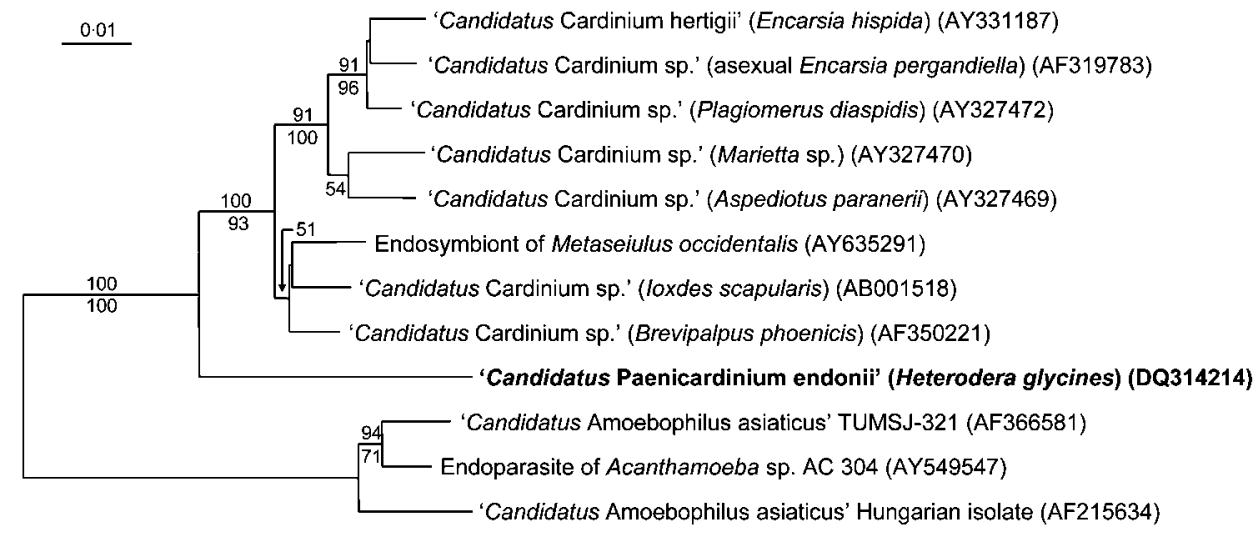

Fig. 3. NJ tree of the bacterial endosymbiont of $H$. glycines, 'Candidatus Paenicardinium endonii', based on HKY85 distances among 16S rRNA gene sequences from selected members of the phylum Bacteroidetes. The tree was rooted using two isolates of 'Candidatus Amoebophilus asiaticus' and an endoparasite of Acanthamoeba sp. as outgroup taxa. The NJ tree is similar to the two trees produced by the MP analysis; numbers above branches indicate bootstrap support (\%) from MP analysis, whereas numbers below branches show bootstrap support (\%) for the NJ topology. Bar, 0.01 substitutions per site. 
discussion focuses only on the DNA-based analyses. The alignment of $g y r B$ gene sequences used comprised 17 taxa and 861 characters, of which 263 were constant and 524 were parsimony-informative. MP analysis yielded two most-parsimonious trees, each 2742 steps long (consistency index 0.43). As was the case with the $16 \mathrm{~S}$ rRNA gene, the two MP trees not only agreed between themselves but they also agreed with the NJ and the ML trees by placing the $H$. glycines endosymbiont in a lineage that had diverged earlier from the branch leading to the bacteria in the recently proposed 'Candidatus Cardinium' (Fig. 4 and Supplementary Fig. S2). Apparently, the speciation event was followed by a more rapid evolution in the branch containing the $H$. glycines endosymbiont than in the one leading to the cardinia. Estimates of nucleotide substitution parameters under the ML criterion, based on the NJ tree, were as follows: $\mathrm{A}-\mathrm{C}, 2 \cdot 36 ; \mathrm{A}-\mathrm{G}, 3 \cdot 75 ; \mathrm{A}-\mathrm{T}$, $1 \cdot 20 ; \mathrm{C}-\mathrm{G}, 2 \cdot 17$; $\mathrm{C}-\mathrm{T}, 7 \cdot 66$; and $\mathrm{G}-\mathrm{T}, 1 \cdot 00$. The proportion of invariant sites (I) was estimated at $0 \cdot 26$ and the shape parameter $(\alpha)$ of the gamma distribution was equal to $1 \cdot 27$.

\section{Proposal of 'Candidatus Paenicardinium endonii'}

The name Paenicardinium (Pae.ni.car.di'ni.um. L. adv. paene nearly, almost; N.L. n. Cardinium the name of an organism in the Candidatus category; N.L. n. Paenicardinium nearly Cardinium) is chosen to acknowledge the morphological similarity between 'Candidatus Paenicardinium' and 'Candidatus Cardinium', both of which contain characteristic arrays of MLSs. The specific epithet endonii (en.do'ni.i. N.L. gen. masc. n. endonii of Endo) is chosen to honour the memory of Dr Burton Yoshiaki Endo, a colleague and friend, who was the first to study this organism.
The status of Candidatus is required by the International Committee on Systematics of Prokaryotes for prokaryotic organisms that are not culturable and thus cannot be characterized according to the International Code of Nomenclature of Bacteria (Murray \& Stackebrandt, 1995). 'Candidatus Paenicardinium endonii' is Gram-negative and rod-shaped and is classified in the phylum Bacteroidetes, class Sphingobacteria, order Sphingobacteriales and family 'Flexibacteraceae' (Garrity \& Holt, 2001).

Two 16S rRNA gene oligonucleotide sequences were reported as unique to 'Candidatus Cardinium' (ZchoriFein et al., 2004), but only the sequence 5'-GGTCTTTAACTGACGCT-3' occurs in the $H$. glycines endosymbiont (position 728). Two additional oligonucleotide sequences, 5'-CGCGATACAATCGTGTGTGTCC-3' (position 822) and 5'-CATCCCTAGAAATAGGGAGTTCCGAAA-3' (position 991), are unique to the bacterium that infects $H$. glycines and, therefore, are diagnostic of 'Candidatus Paenicardinium endonii'. The $\mathrm{G}+\mathrm{C}$ content of the $16 \mathrm{~S}$ rRNA gene of the bacterium is $52 \mathrm{~mol} \%$, compared with $48 \mathrm{~mol} \%$ for 'Candidatus C. hertigii' (Zchori-Fein et al., 2004). 'Candidatus Paenicardinium endonii' shares $94 \%$ 16S rRNA gene nucleotide sequence identity with the endosymbiont of Metaseiulus occidentalis and $93 \%$ identity with 'Candidatus C. hertigii'. The gyrB nucleotide sequence shares $83 \%$ identity with the 'Candidatus Cardinium' endosymbiont of Encarsia pergandiella and $81 \%$ identity with 'Candidatus C. hertigii'. These levels of identity for the $16 \mathrm{~S}$ rRNA and gyrB genes are indicative of a distinct taxon (Ludwig et al., 1998). The name 'Candidatus Paenicardinium endonii' is proposed on the basis of the 16S rRNA gene sequence (GenBank accession no. DQ314214), the gyrB gene sequence (accession no. DQ314215), the signature sequences listed above and the presence of MLSs. The bacterium found in the population of H. glycines from the Ames Plantation near Grand Junction, TN, USA, is the proposed type strain.

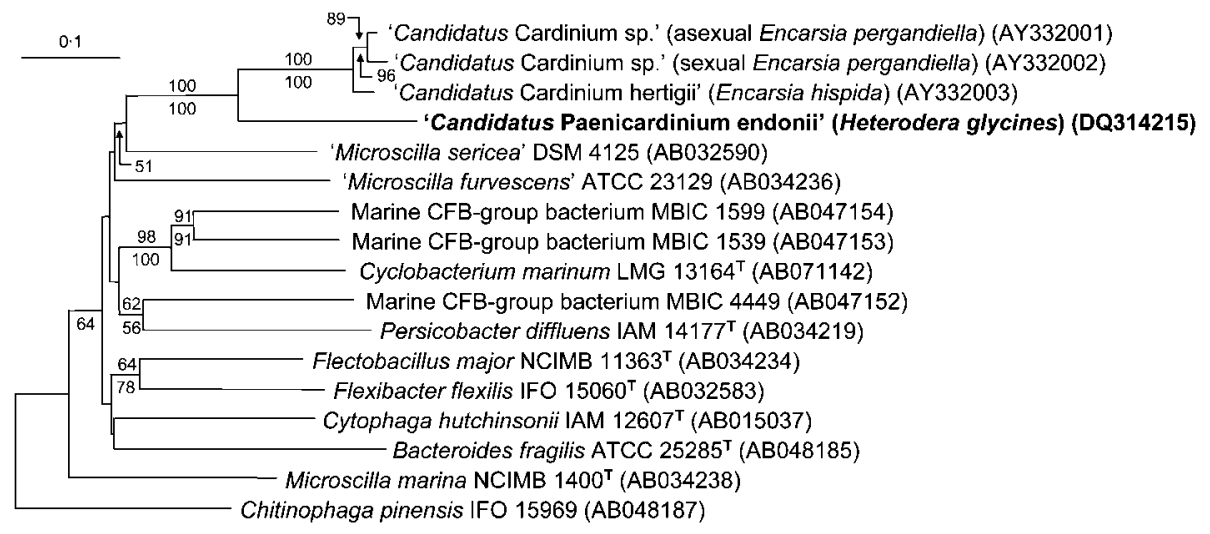

Fig. 4. $\mathrm{NJ}$ tree of the bacterial endosymbiont of $H$. glycines, 'Candidatus Paenicardinium endonii', based on HKY85 distances among $g y r B$ gene sequences from selected members of the phylum Bacteroidetes. The tree was rooted using the bacterium Chitinophaga pinensis IFO 15969 as the outgroup taxon. The NJ tree is similar to the two trees produced by MP analysis; numbers above branches indicate bootstrap support (\%) from MP analysis, whereas numbers below branches show bootstrap support (\%) for the $\mathrm{NJ}$ topology. Bar, $0 \cdot 1$ substitutions per site. 


\section{ACKNOWLEDGEMENTS}

The authors thank Barbara Stanger for technical assistance with the electron microscopy. Mention of trademark or proprietary products does not constitute a guarantee or warranty of the products by the US Department of Agriculture and does not imply their approval to the exclusion of other products that may also be suitable.

\section{REFERENCES}

Altschul, S. F., Madden, T. L., Schäffer, A. A., Zhang, J., Zhang, Z., Miller, W. \& Lipman, D. J. (1997). Gapped BLAST and PSI-BLAST: a new generation of protein database search programs. Nucleic Acids Res 25, 3389-3402.

Ashelford, K. E., Weightman, A. J. \& Fry, J. C. (2002). PRIMROSE: a computer program for generating and estimating the phylogenetic range of $16 \mathrm{~S}$ rRNA oligonucleotide probes and primers in conjunction with the RDP-II database. Nucleic Acids Res 30, 3481-3489.

Atibalentja, N., Noel, G. R. \& Ciancio, A. (2004). A simple method for PCR-amplification, cloning, and sequencing of Pasteuria 16S rDNA from small numbers of endospores. J Nematol 36, 100-105.

Cole, J. R., Chai, B., Marsh, T. L. \& 8 other authors (2003). The Ribosomal Database Project (RDP-II): previewing a new autoaligner that allows regular updates and the new prokaryotic taxonomy. Nucleic Acids Res 31, 442-443.

Endo, B. Y. (1979). The ultrastructure and distribution of an intracellular bacterium-like microorganism in tissues of larvae of the soybean cyst nematode, Heterodera glycines. J Ultrastruct Res 67, $1-14$.

Garrity, G. M. \& Holt, J. G. (2001). The road map to the Manual. In Bergey's Manual of Systematic Bacteriology, 2nd edn, vol. 1, pp. 119-166. Edited by D. R. Boone, R. W. Castenholz \& G. M. Garrity. New York: Springer.

Hanaichi, T., Sato, T., Iwamoto, T., Malavasi-Yamashiro, J., Hoshino, M. \& Mizuno, N. (1986). A stable lead by modification of Sato's method. J Electron Microsc 35, 304-306.

Kaya, H. K. (1993). Entomogenous and entomopathogenic nematodes in biological control. In Plant Parasitic Nematodes in Temperate Agriculture, pp. 565-591. Edited by K. Evans, D. L. Trudgill \& J. M. Webster. Wallingford, UK: CAB International.
Lane, D. J. (1991). 16S/23S rRNA sequencing. In Nucleic Acid Techniques in Bacterial Systematics, pp. 115-175. Edited by E. Stackebrandt \& M. Goodfellow. New York: Wiley.

Ludwig, W., Strunk, O., Klugbauer, S., Klugbauer, N., Weizenegger, M., Neumaier, J., Bachleitner, M. \& Schleifer, K. H. (1998). Bacterial phylogeny based on comparative sequence analysis. Electrophoresis 19, 554-568.

Maddison, D. R. \& Maddison, W. P. (2001). MacClade version 4.0.1. Sunderland, MA: Sinauer Associates.

Murray, R. G. E. \& Stackebrandt, E. (1995). Taxonomic note: implementation of the provisional status Candidatus for incompletely described procaryotes. Int J Syst Bacteriol 45, 186-187.

Saint André, A. V., Blackwell, N. M., Hall, L. R. \& 9 other authors (2002). The role of endosymbiotic Wolbachia bacteria in the pathogenesis of river blindness. Science 295, 1892-1895.

Shepherd, A. M., Clark, S. A. \& Kempton, A. (1973). An intracellular micro-organism associated with tissues of Heterodera spp. Nematologica 19, 31-34.

Swofford, D. L. (2003). PAUP* - Phylogenetic Analysis Using Parsimony ${ }^{\star}$ and other methods, version 4. Sunderland, MA: Sinauer Associates.

Taylor, M. J. (2003). Wolbachia in the inflammatory pathogenesis of human filariasis. Ann N Y Acad Sci 990, 444-449.

Thompson, J. D., Gibson, T. J., Plewniak, F., Jeanmougin, F. \& Higgins, D. G. (1997). The CLUSTAL_X windows interface: flexible strategies for multiple sequence alignment aided by quality analysis tools. Nucleic Acids Res 25, 4876-4882.

Vandekerckhove, T. T. M., Willems, A., Gillis, M. \& Coomans, A. (2000). Occurrence of novel verrucomicrobial species, endosymbiotic and associated with parthenogenesis in Xiphinema americanumgroup species (Nematoda, Longidoridae). Int J Syst Evol Microbiol 50, 2197-2205.

Yamamoto, S. \& Harayama, S. (1995). PCR amplification and direct sequencing of $\operatorname{gyr} B$ genes with universal primers and their application to the detection and taxonomic analysis of Pseudomonas putida strains. Appl Environ Microbiol 61, 1104-1109.

Zchori-Fein, E., Perlman, S. J., Kelly, S. E., Katzir, N. \& Hunter, M. S. (2004). Characterization of a 'Bacteroidetes' symbiont in Encarsia wasps (Hymenoptera: Aphelinidae): proposal of 'Candidatus Cardinium hertigii’. Int J Syst Evol Microbiol 54, 961-968. 